\title{
Acute multiple focal neuropathies and delayed postanoxic encephalopathy after alcohol intoxication
}

\section{Wei-Che Wang \\ Hsiu-Chun Yang \\ Yao-Jen Chen}

Department of Physical Medicine and Rehabilitation, Chung Shan Medical University Hospital, Taichung, Taiwan
This article was published in the following Dove Press journal:

Neuropsychiatric Disease and Treatment

17 July 2015

Number of times this article has been viewed
Correspondence: Yao-Jen Chen Department of Physical Medicine and Rehabilitation, Chung Shan Medical University Hospital, I I 0 section I ChienKuo North Road, Taichung 402, Taiwan

$\mathrm{Tel}+886424739595$ ext 2I 700

Fax $+8864247 \mid 0934$

Email yj.chen1960@gmail.com
Abstract: Acute-onset alcohol-associated neuropathy is only occasionally reported, and delayed postanoxic encephalopathy is rare. Here, we report a male who developed acute multiple focal neuropathies and later delayed postanoxic encephalopathy after alcohol intoxication. He had hypoxia and rhabdomyolysis, presenting with acute renal failure initially, and cardiopulmonary support, including mechanical ventilation, led to improvement of the patient at the acute stage. He suffered from bilateral hand numbness and mild weakness of the right lower limb thereafter. Nerve-conduction study revealed no pickup of compound muscle action potential or sensory nerve action potential in the bilateral ulnar nerve, but showed attenuated amplitude of compound muscle action potential in the right femoral nerve. Multiple focal neuropathies were suspected, and he received outpatient rehabilitation after being discharged. However, the patient developed gradual onset of weakness in four limbs and cognitive impairment 23 days after the hypoxia event. Brain computed tomography showed low attenuation over bilateral globus pallidus, and brain magnetic resonance imaging disclosed diffuse increased signal intensity on $T_{2}$-weighted images and fluid-attenuated inversion recovery in bilateral white matter. He was admitted again under the impression of delayed postanoxic brain injury. Supportive treatment and active rehabilitation were given. He had gradual improvement in motor and functional status after rehabilitation. He could walk with festinating gait under supervision, and needed only minimal assistance in performing activities of daily living approximately 1 year later.

Keywords: multiple focal neuropathies, delayed postanoxic encephalopathy, alcohol intoxication, rhabdomyolysis

\section{Introduction}

Acute alcohol intoxication can lead to harmful effects on different organs and apparatuses, such as behavior, cardiac, gastrointestinal, pulmonary, neurological, and metabolic systems. ${ }^{1}$ Symptoms are usually related to blood alcohol concentration. At a blood alcohol concentration higher than $300 \mathrm{mg} / \mathrm{dL}$, there is increased risk of respiratory depression and arrest. Alcohol-associated neuropathies are mostly of insidious onset, but there are acute forms of neuropathies occasionally. ${ }^{2}$ Hypoxiaassociated encephalopathy is mostly of acute form, but there are rare occurrences of delayed forms of postanoxic encephalopathy. ${ }^{3,4}$ We report a 45 -year-old male who developed myoglobinuric renal failure, acute multiple focal neuropathies, and delayed postanoxic encephalopathy after alcohol intoxication.

\section{Case report}

A 45-year-old male denied any systemic disease before, but reported sometimes drinking alcohol with his friends for approximately 20 years. The patient drank two bottles 
of whiskey with his friends in the evening on the day of what happened. His wife found the patient lying on the sofa at midnight. Because the patient was found to be in unconscious status for approximately 12 hours, he was sent to a medical center in mid-Taiwan by an emergency medical technician. The initial technician's record revealed decreased oxygen saturation $(55 \%)$ by pulse oximetry, tachycardia (heart rate 110 bpm), and hypotension (blood pressure 90/72 mmHg). At the emergency department, Glasgow Coma Scale results were eye response 1, verbal response 1 , and motor response 1 . Blood-gas analysis demonstrated hypoxemia (partial pressure of oxygen $49 \mathrm{mmHg}$ and partial pressure of carbon dioxide $45 \mathrm{mmHg}$ ). Emergent intubation was done, and computed tomography of the brain showed poor demarcation of the gray-white matter junction, mild sulcus effacement, and no evidence of hemorrhagic lesion (Figure 1, A and B). Other laboratory investigations demonstrated elevated plasma creatine kinase (1,268 IU/L, laboratory reference 40-280 IU/L), elevated plasma alcohol concentration (364 mg/dL), and elevated plasma creatinine $(3.7 \mathrm{mg} / \mathrm{dL}$, laboratory reference $0.7-1.3 \mathrm{mg} / \mathrm{dL}$ ). Levels of benzodiazepine, monoxide, and ammonia were within normal limits. He was admitted to the intensive care unit under the impression of acute respiratory failure due to acute alcohol intoxication and rhabdomyolysis complicated with acute renal failure for further management. His consciousness gradually recovered after mechanical ventilation and adequate hydration. Thereafter, successful extubation was done. At day 5, he was transferred to an ordinary ward. Numbness over bilateral hands and mild weakness of the right lower limb were complained of at the ordinary ward. Magnetic resonance imaging (MRI) of the thoracolumbar spine was done, but did not reveal specific findings. Nerve-conduction study (NCS) showed no pickup of compound muscle action potential (CMAP), sensory nerve action potential over bilateral ulnar nerves, and attenuated amplitude of CMAP in the right femoral nerve. As he denied any sensory impairment or motor weakness before this episode, acute multiple focal neuropathies were suspected. At day 15, he was discharged with clear consciousness, and he was able to perform all activities of daily living (ADL) independently. He received outpatient rehabilitation for multiple focal neuropathies. Unfortunately, involuntary movement over bilateral upper extremities and weakness of bilateral lower limbs were noted at day 25 after the hypoxic event. He was unable eat by himself and could not communicate with his family. Impairment of sphincter control with urine and stool incontinence was also found. Brain computed tomography showed focal low-attenuation lesions in the bilateral globus pallidus.
Brain MRI demonstrated diffuse increased signal intensity on $T_{2}$-weighted imaging (T2WI) and fluid-attenuated inversion recovery (FLAIR) in bilateral white matter (Figure 1, C and D). Owing to a history of hypoxia and the findings of the brain MRI, he was admitted under the tentative diagnosis of delayed postanoxic encephalopathy. Minimal mental status examination was done, and the score was 4 . He was only able to obey little one-step orders, and had little verbal expression. He was dependent in all basic ADL. We prescribed supportive managements, such as chest care and changing position frequently, to prevent complications. Rehabilitation training programs focusing on joint range-of-motion exercise, muscle strength, endurance training, basic ADL training, and cognitive-behavioral training were also arranged. Gradual improvement in motor function and cognitive state was noted. He continued to receive outpatient rehabilitation after being discharged. The patient showed improvement in motor and cognitive function little by little. Atrophy in intrinsic muscles of the bilateral hands and the right thigh muscle were still noted at day 90. NCS and electromyography was done for follow-up. NCS still showed no CMAP or sensory nerve action potential in left ulnar nerve, but there was attenuated CMAP in right ulnar nerve. Also, attenuated CMAP of the right femoral nerve was found. Electromyography displayed polyphasic waves with decreased recruitment in the right second dorsal interosseus muscle and right rectus femoris muscle. There was no motor-unit action potential of the left second dorsal interosseus muscle. Brain MRI was executed again at day 95 after the hypoxic event, and revealed less increased signal intensity on T2WI and FLAIR in bilateral white matter compared to previous study (Figure 1, E and F). He could walk by himself in a festinating gait and perform basic ADL under minimal assistance approximately 1 year after the event. He continued to be followed up until 6 years posthypoxia. Brain MRI still demonstrated increased signal intensity over bilateral white matter on T2WI and FLAIR, but the extent was less than 6 years previously. Atrophy of the brain was also noted (Figure 1, G and H). During these years, we prescribed dihydroergotoxine mesylate $1.5 \mathrm{mg}$ three times per day for remnant cognitive impairment and levodopa $200 \mathrm{mg} /$ benserazide $50 \mathrm{mg}$ twice daily for shuffling gait. Now, he was totally independent in basic ADL, but still had the festinated gait pattern.

\section{Discussion}

Alcohol intoxication can result in a series of organ dysfunctions. We report a case of acute multiple focal neuropathies and delayed postanoxic encephalopathy after alcohol 

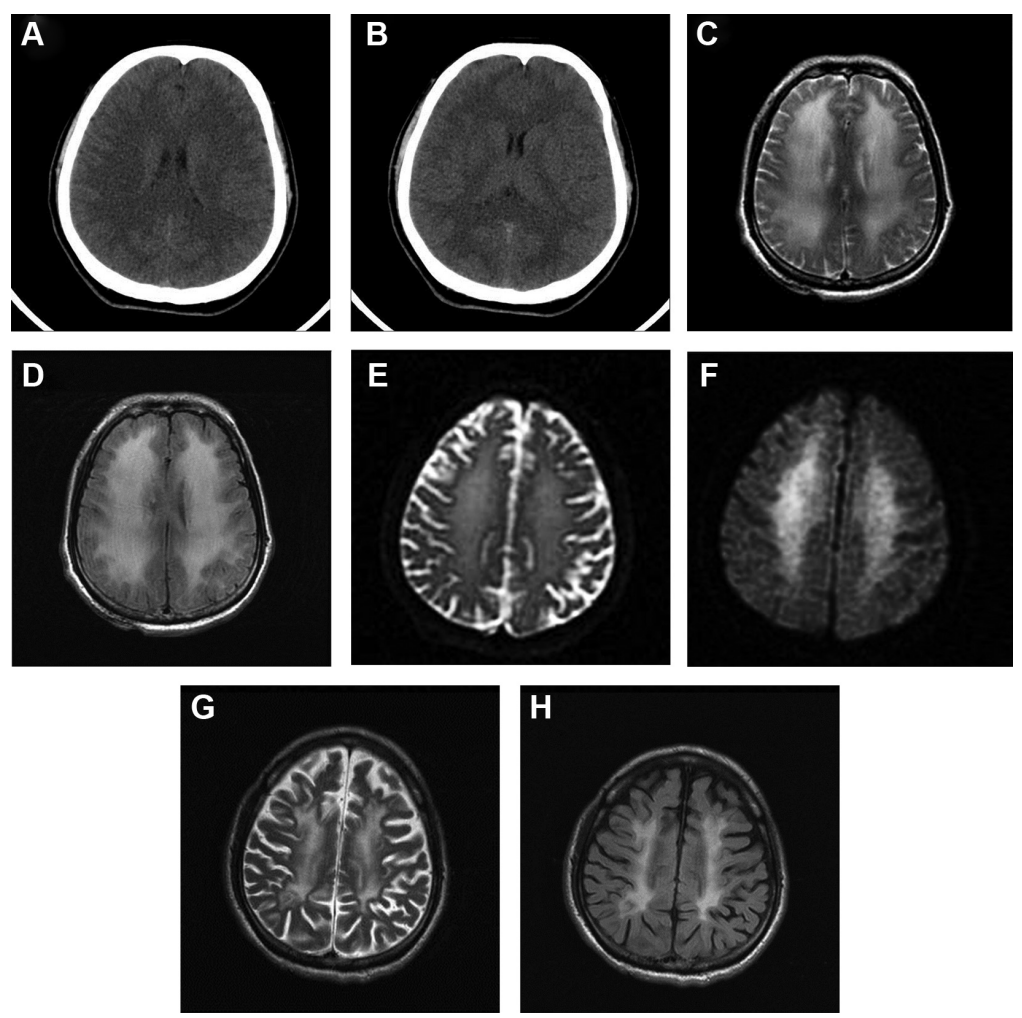

Figure I Images of delayed postanoxic encephalopathy.

Notes: (A and B) Brain CT at day I; image A shows a higher axial cut level than image $\mathbf{B}$ which shows an axial cut at the basal ganglion level; (C) T2WI of brain MRI at day 30; (D) FLAIR of brain MRI at day 30; (E) T2WI of brain MRI at day 95; (F) FLAIR of brain MRI at day 95; (G) T2WI of brain MRI 6 years after the hypoxic event; (H) FLAIR of brain MRI 6 years after the hypoxic event.

Abbreviations: CT, computed tomography; T2WI, $T_{2}$-weighted imaging; MRI, magnetic resonance imaging; FLAIR, fluid-attenuated inversion recovery.

intoxication. Most alcohol-induced neuropathy is chronic distal symmetrical polyneuropathy with initially insidious development of sensory symptoms. However, there is infrequently occurrence of an acute course of polyneuropathy in patients with chronic alcohol use. ${ }^{2}$ The interval from the onset of symptoms to the nadir ranges from a few days to several weeks. The manifestations of symptoms are due to both sensory and motor-nerve involvement. These forms of neuropathy are described as axonal involvement on electrophysiological and historical examination. Our case presented as acute onset of multiple focal neuropathies; possible etiologies included compartment syndrome following rhabdomyolysis, direct toxic effect of alcohol, or limb compression after drinking. Jeon et $\mathrm{al}^{5}$ reported a case of lumbosacral plexopathy due to rhabdomyolysis in the hip muscle after alcohol consumption. The diagnosis was made by MRI of the pelvis, which showed abnormal signal intensity and heterogeneous enhancement in both gluteal muscles. Anwar et $\mathrm{al}^{6}$ described a case of bilateral brachial plexus injuries after drinking. The cause was postulated to be that the patient had prolonged immobilization in hyperabducted posture of the arms after drinking alcohol. Wang et $\mathrm{al}^{7}$ related a case of rhabdomyolysis and multiple focal neuropathies after drinking alcohol soaked with centipede. The involved nerves were left median, left ulnar, and right radial. There have been reports of toxic effects of alcohol to nerves instead of nutritional neuropathy caused by thiamine deficiency. ${ }^{8,9}$ The pattern of involvement of multiple focal neuropathies in the present case was bilateral ulnar nerves and right femoral nerve. The most probable etiology was a direct toxic effect of alcohol. Compartment syndrome or limb compression are less likely. The patient had gradual recovery of atrophy of intrinsic muscles of bilateral hands and right quadriceps.

Delayed postanoxic encephalopathy is a neurological deterioration that occurs in patients some time after they have recovered from initial anoxia or hypoxia. ${ }^{3,4}$ Incidence is reported to range from less than one to 28 per 1,000 patients who have suffered an anoxic or hypoxic event. The interval between anoxic or hypoxic event and abrupt neurological decline usually ranges from 1 to 4 weeks or more. The interval for the present case was approximately 25 days. The most common cause of delayed postanoxic encephalopathy is carbon monoxide exposure, but other 
anoxic events have also been reported. Autopsy reports often reveal diffuse demyelination of subcortical white matter, but the exact pathogenesis is not clear. While comprehensive physical examination and history taking is necessary in diagnosing delayed postanoxic encephalopathy, MRI can be very helpful in diagnostic workup. The prognosis of delayed postanoxic encephalopathy is still not clear. Rehabilitation for delayed postanoxic encephalopathy can prevent complications, such as immobilization, and can improve patients' functional status. The present case had recovery of motor function step by step, and he was able to perform basic ADL under minimal assistance 1 year later. However, he still had impaired cognition, and did not return to work until recently.

\section{Conclusion}

Most alcohol-associated neuropathies are of insidious onset, but there are acute forms of neuropathies occasionally. Most hypoxic encephalopathy is of acute form. However, there are rare occurrences of delayed postanoxic encephalopathy. Clinicians should be aware of these for differential diagnoses that would lead to correct diagnosis for appropriate management and avoid unnecessary diagnostic workup. The prognosis of postanoxic encephalopathy is not clear, but rehabilitation programs may be beneficial for the promotion of functional status.

\section{Acknowledgment}

The authors report that at their institute (Chung Shan Medical University Hospital, Taichung, Taiwan), institutional review board approval for a single case report is not required.

\section{Disclosure}

The authors report no conflicts of interest in this work.

\section{References}

1. Vonghia L, Leggio L, Ferrulli A, Bertini M, Gasbarrini G, Addolorato G. Acute alcohol intoxication. Eur J Intern Med. 2008;19(8):561-567.

2. Wöhrle JC, Spengos K, Steinke W, Goebel HH, Hennerici M. Alcoholrelated acute axonal polyneuropathy: a differential diagnosis of GuillainBarré syndrome. Arch Neurol. 1998;55(10):1329-1334.

3. Goedee S, van der Nat GA, Roks G. Temporary recovery after resuscitation: delayed postanoxic encephalopathy. BMJ Case Rep. Epub 2013 Apr 15.

4. Custodio CM, Basford JR. Delayed postanoxic encephalopathy: a case report and literature review. Arch Phys Med Rehabil. 2004; 85(3):502-505.

5. Jeon HJ, Cho BM, Oh SM, Park SH. Lumbosacral plexopathy, complicating rhabdomyolysis in a 57-year-old man, presented with sudden weakness in both legs. J Korean Neurosurg Soc. 2007;42(6):481-483.

6. Anwar F, McLaughlin D, Panesar BS. Bilateral brachial plexus injury. J Coll Physicians Surg Pak. 2012;22(3):176-178.

7. Wang IK, Hsu SP, Chi CC, et al. Rhabdomyolysis, acute renal failure, and multiple focal neuropathies after drinking alcohol soaked with centipede. Ren Fail. 2004;26(1):93-97.

8. Mellion M, Gilchrist JM, de la Monte S. Alcohol-related peripheral neuropathy: nutritional, toxic, or both? Muscle Nerve. 2011;43(3):309-316.

9. Koike H, Iijima M, Sugiura M, et al. Alcoholic neuropathy is clinicopathologically distinct from thiamine-deficiency neuropathy. Ann Neurol. 2003; 54(1):19-29.

\section{Publish your work in this journal}

Neuropsychiatric Disease and Treatment is an international, peerreviewed journal of clinical therapeutics and pharmacology focusing on concise rapid reporting of clinical or pre-clinical studies on a range of neuropsychiatric and neurological disorders. This journal is indexed on PubMed Central, the 'PsycINFO' database and CAS, and is the official journal of The International Neuropsychiatric Association (INA). The manuscript management system is completely online and includes a very quick and fair peer-review system, which is all easy to use. Visit http://www.dovepress.com/testimonials.php to read real quotes from published authors. 